Projets

de paysage

\section{Projets de paysage}

Revue scientifique sur la conception et l'aménagement de l'espace

\section{$21 \mid 2019$}

Paysage en action sous les tropiques

\title{
Les aires marines protégées du Sud-Ouest malgache
}

Des paysages sacrés des pêcheurs vezo aux paysages désincarnés des agents de la conservation

Protected Marine Areas in Southwestern Madagascar - From the Sacred Landscapes of the Vezo Fishermen to the Intangible Landscapes of

Conservationists

\section{Francis Veriza et Laurent Couderchet}

\section{OpenEdition}

\section{Journals}

Édition électronique

URL : http://journals.openedition.org/paysage/2447

DOI : 10.4000/paysage.2447

ISSN : 1969-6124

\section{Éditeur :}

École nationale supérieure du paysage de Versailles-Marseille, Institut national des sciences appliquées Centre Val de Loire - École de la nature et du paysage, École nationale supérieure d'architecture et de paysage de Bordeaux, École nationale supérieure d'architecture et de paysage de Lille, Agrocampus Angers

\section{Référence électronique}

Francis Veriza et Laurent Couderchet, "Les aires marines protégées du Sud-Ouest malgache », Projets de paysage [En ligne], 21 | 2019, mis en ligne le 30 décembre 2019, consulté le 28 février 2020. URL : http://journals.openedition.org/paysage/2447 ; DOI : 10.4000/paysage.2447 


\section{Les aires marines protégées du Sud- Ouest malgache}

Des paysages sacrés des pêcheurs vezo aux paysages désincarnés des agents de la conservation

Protected Marine Areas in Southwestern Madagascar - From the Sacred Landscapes of the Vezo Fishermen to the Intangible Landscapes of

Conservationists

Francis Veriza et Laurent Couderchet

1 Notre sujet est tiré d'une recherche doctorale qui concerne la mise en ressource des milieux du littoral sud-ouest malgache et l'effet de cette mise en ressource sur le rapport au monde de la population locale (Veriza, 2019). La question du paysage n'est pas centrale dans la thèse qui nous sert de support, nous la mettons au cœur de la réflexion pour cette contribution à la revue Projet de paysage. Sur le plan méthodologique, cela revient à remobiliser un matériau existant pour une analyse nouvelle à travers le prisme du paysage.

2 La société vezo occupe le littoral sud-ouest de Madagascar, son identité est fondée sur son lien à la mer et son activité de pêche. Peut-on considérer le paysage en pays vezo ? Les Vezo n'ont pas de mot pour dire paysage. Leur vocabulaire est pourtant d'une grande richesse pour désigner leur milieu. La mer a un dos ambohone, un ventre antsodrano, une bouche vavarano, une lèvre sonindriake, une tête lohariake. La traduction est un des instruments de la méthode, elle est lexicale mais aussi conceptuelle. La recherche est effectuée depuis le dedans. Nous observons la perturbation de la cosmophanie vezo sous l'effet de la mise en place des aires marines protégées, «la perte de cosmicité qu'entraîne le divorce entre monde sensible (la cosmophanie vezo) et monde intelligible (le zonage de la science moderne, celui des aires marines protégées) ", la transformation du paysage depuis le dehors en "vérité physique... espace froid de la raison calculante » (Berque, 1998).

3 Notre terrain est constitué par la bande littorale depuis le Sud de Toliara jusqu'à la région de Morombe au nord, soit plus de 250 kilomètres linéaires. Cette grande emprise 
permet de traduire le phénomène de mobilité des villages dont les constructions légères se déplacent avec les stratégies des pêcheurs, elle permet aussi d'observer le partage du territoire entre les organisations de développement et de conservation: d'une part la mobilité, d'autre part l'ancrage. L'étude du grand linéaire est complétée par une analyse en immersion de la situation dans les environs d'Andavadoake, à 25 kilomètres au sud de Morombe.

4 L'observation des transformations sociospatiales que nous présentons s'est effectuée sur une quinzaine d'années, elle prend la forme d'une immersion ethnogéographique longue et d'entretiens étalés dans le temps parfois avec les mêmes interlocuteurs, pêcheurs non impliqués, acteurs des projets de développement et de conservation, notables, qui changent de discours selon les contextes, les opportunités et les jeux de pouvoir. Le matériau - entretiens, observations participantes - a été réuni initialement pour une exploitation non paysagère. L'hypothèse que nous proposons ici est qu'il peut redire des choses, différemment, si le paysage en devient l'outil d'analyse. À Andavadoake, le temps de l'immersion nous permet d'estimer l'évolution de l'occupation de l'espace ainsi que celle des pratiques de pêche, dont l'originalité était la gestion des tanifaly, sites sacrés. La recherche a été l'occasion d'assister à la mise en place du réseau d'aires marines protégées Velondriake sous pilotage des ONG Blue Venture et WCS.

5 Les Vezo sont considérés comme une ethnie, dans les limites d'un territoire. L'histoire du peuplement montre pourtant qu'il n'y a pas d'origine vezo mais une communauté de pratiques, de représentations de l'espace et de croyances, un être au monde. Les Vezo sont ouverts à l'extérieur, leur espace est accessible aux gens de la terre massikoro, par exemple. Nous proposons la notion de "vezoification " pour désigner l'incorporation progressive dans la société vezo d'individus ou de groupes non originaires du littoral mais qui en adoptent les pratiques, les croyances et les règles. Le dispositif de réserves marines Velondriake que nous étudions correspond à une rupture dans ce mouvement. Il justifie une intervention étrangère qui impose ses règles et son rapport au paysage : la mer n'est plus sacrée mais ressource, réservoir de biodiversité, son accès est contrôlé ; l'espace cesse d'être vezo pour devenir vazaha, les Vazaha étant les étrangers de l'extérieur, notamment européens, qui continuent d'imposer leurs règles, désormais écomondialistes.

6 Dans un premier temps, nous développons la notion de vezoïfication, adoption d'un rapport au monde de la mer. Nous présentons ensuite le milieu des Vezo sous l'angle de la cosmophanie, les mots vezo étant instruments de compréhension. Les programmes de développement et de conservation se sont traduits par la désorganisation du système originel de préservation de la ressource, fait de parcimonie des prises et d'interdits concernant la fréquentation d'espaces de reconstitution des stocks. Nous restituons le cycle développement-conservation dans un troisième temps avant d'étudier la dévezoïfication et de proposer l'idée d'un cosmocide pour traduire le déclin du groupe vezo dans ses caractéristiques originales.

\section{Vezoïfication, être au monde}

7 Les Vezo sont des pêcheurs semi-nomades du littoral sud-ouest de Madagascar qui se distinguent des agriculteurs et éleveurs de l'intérieur. Ils constituent officiellement une des dix-huit ethnies de Madagascar. Le répertoire des groupes ethniques varie d'une 
époque à l'autre, d'un auteur à l'autre. Ainsi, en 1905, les administrateurs coloniaux recensaient vingt-cinq groupes ethniques sur l'île, ils n'étaient plus que dix-huit à la veille de l'indépendance (Rajaonarison cité par Razafindrazaka, 2010). Le premier président de la République, Philibert Tsiranana, insistait sur la présence d'un dixneuvième groupe, connu sous le nom générique "vazaha »: le colonisateur ainsi que tous ceux qui étaient venus à Madagascar pour raison de commerce depuis le $\mathrm{XIX}^{\mathrm{e}}$ siècle. L'approche homogénéisante ethnique est reprise de la colonisation par l'État malgache dès l'indépendance et utilisée jusqu'à aujourd'hui.

«Sous la colonisation et dans le cadre de la politique ethnique du général Gallieni, $1^{\text {er }}$ gouverneur français de Madagascar, l'unification du territoire s'est effectuée par le truchement de la transformation des identités territoriales qui ont imposé leurs noms aux populations qui occupaient ces territoires » (Veriza et al., 2018).

Il s'agissait de mieux contrôler le territoire et les populations afin d'asseoir la domination coloniale (Dina, 1982). L'ethnie est bien ici un instrument idéologique et politique (Retaillé, 2003) ; c'est l'affirmation naturalisée d'un groupe originel enfermé dans des limites territoriales. Mais peut-on considérer un territoire vezo avec ses bornes alors que ce sont la mobilité et l'horizon marin qui caractérisent son espace?

9 L'histoire généalogique des groupes sociaux et celle de leurs migrations locales (Veriza, 2019) permettent d'identifier les nombreuses stratégies destinées à conquérir un statut dans les ordres précolonial, colonial et enfin malgache. Elles remettent en cause les classifications ethniques fixées par l'administration pour de nouveaux ordonnancements sociospatiaux. Selon Marikandia (1988), le mot «vezo" était à l'origine un nom de plaisanterie donné par les voisins chasseurs-cueilleurs de l'intérieur, les Velognala. Étymologiquement, vezo serait l'impératif du verbe mivé, littéralement : "pagayer » ou " ramer ». «Vezo (malaky) lehiroa laka io!», " pagaie (vite) cette pirogue! », l'expression est fréquemment utilisée pour désigner cette population et suggère la menace; en cas de danger sur terre, un Vezo peut prendre la fuite avec sa famille à l'aide de sa pirogue. Le mot lay, "voile » en traduction libre, signifie " fuite " dans sa traduction littérale. Cette nuance linguistique caractérise le tempérament vezo.

«Maintes fois, jadis, la mer a été le refuge des "Vezo" contre les incursions des

"Masikoro". Ils se jetaient à l'eau sur leurs pirogues rapides pour sauver leur vie et

leur liberté » (Engelvin, 1937).

On peut alors considérer les Vezo comme des « orphelins de la terre ", installés au bord de la mer ou sur les îlots avec les Velondriake, enfants de la mer. Les Vezo se déplacent avec une pirogue à balancier le long de la côte sud-ouest pour préserver leur liberté mais aussi pour suivre le banc de poissons. Un vieux pêcheur vezo d'Andavadoake explique :

« Nous ne pouvons pas vivre sans la mer. Nous avons intérêt de ne pas transgresser les interdits sinon elle sera en colère contre nous. À la première mise à l'eau d'un nouveau filet ou d'une nouvelle pirogue, nous devons effectuer une cérémonie de demande de bénédiction aux ancêtres et aux esprits en mer pour éviter d'éventuels accidents » (propos recueilli le 28 avril 2006).

11 C'est ainsi que le Vezo a pu fonder son tanindraza qui traverse les deux royaumes maroserana et andrevola le long de la côte sud-ouest. Ce tanindraza a été construit, démantelé, déplacé, reconstruit au gré des événements. Déjà la fondation du tanindraza était complexe, en lien avec la stratégie de mobilité pour éviter les relations avec les dominants. Généralement, il est difficile pour un vahiny, c'est-à-dire un étranger, de s'installer dans un territoire dont il n'est pas originaire. Chez les Vezo, l'intégration se 
déroule facilement en respectant les règles imposées par les tompontanà, les maitres du village.

«Le territoire est la prison que les hommes se donnent »(Raffestin, 1980), les Vezo sont semi-nomades sans souverains à la différence des agriculteurs de l'intérieur (Veriza et al., 2018). Avant d'être territoire, le littoral sud-ouest est milieu, au sens d'Augustin Berque (1998), pour un peuplement humain mobile et mouvant dans ses contours, front en construction permanente, horizon. La mobilité des hommes à l'intérieur de limites floues est une force et une faiblesse face aux différentes colonisations. L'espace des Vezo autoconstruit se distingue ainsi du territoire qui leur est assigné depuis l'extérieur par les différentes puissances dominantes. L'endorégulation se manifeste par la vézoïfication : constitution d'une identité collective fondée sur l'exploitation de la mer qui devient mère.

13 La spécificité vezo associant la marge, la navigation et la pêche est admise par toute la communauté des chercheurs, nationaux ou étrangers, travaillant sur le littoral sudouest de Madagascar. L'espace vezo est mobile au sens de Retaillé (2005). Les pouvoirs successifs précoloniaux, coloniaux et postcoloniaux ont tenté d'enfermer le groupe dans des limites, un territoire dont il n'a cessé de s'échapper. Le mouvement actuel d'enfermement de la nature (Amelot et al., 2014) est la reproduction d'assignations antérieures. Comme les précédentes, cette assignation fait violence à l'organisation sociospatiale.

\section{Cosmophanie}

La notion de paysage n'existe pas dans toutes les cultures, prétend Augustin Berque $(1998,2019)$. Il précise que pour les sciences de la nature, le paysage étant la forme de l'environnement, il y a partout et toujours paysage. Il oppose ainsi une approche phénoménologique en sciences humaines à l'approche positiviste des sciences de la nature.

« Du moment qu'une certaine relation s'instaure nécessairement entre les sociétés humaines et l'environnement qu'elles habitent, et du moment que la notion de paysage n'est pas universelle, un concept plus général que la notion de "paysage" s'impose donc pour qualifier le côté sensible de ladite relation, c'est-à-dire le fait que l'environnement apparaît toujours d'une certaine façon aux diverses sociétés humaines. Ce concept, c'est celui de cosmophanie, autrement dit la manière dont le monde (kosmos) se manifeste (phainei) à telle ou telle société. [...] La cosmophanie est un concept mésologique, ce qui signifie que ce qui apparaît à un certain être - ce qui est la réalité pour cet être - n'est pas l'environnement lui-même, mais une certaine relation à l'environnement ; à savoir le milieu qui est propre à cet être " (Berque, 2019).

15 En pays vezo s'affrontent les deux conceptions du paysage : le rapport au monde d'une "civilisation autre que paysagère » et le paysage extrait du milieu, celui de la "décosmisation moderne» (Berque, 1998). Si l'on accepte cette conception du milieu, on admet la difficulté qu'il peut y avoir à concevoir un programme de conservation du milieu vezo depuis Londres, Londres étant le siège de l'ONG Blue Ventures qui pilote le programme d'aires marines protégées pour les communautés locales vezo à Andavadoake.

16 L'incarnation des Vezo dans leur milieu a donné naissance à un certain nombre de coutumes et de traditions qui visent à la fois à mieux gérer les ressources et à maintenir 
la cohésion sociale. Les Vezo sont à la fois des «enfants de la mer» (Engelvin, 1937), " prédateurs du platier » ou « semi-nomades » (Koechlin, 1975), lambondriake « sangliers de la mer ", tompondriake "maîtres de la mer ", ou encore des masondrano " yeux de la mer» (Rejela, 1993), «marins-pêcheurs" (Cormier-Salem, 1995). Toutes ces qualifications correspondent au caractère idéologique et social du groupe. Après avoir perdu leurs droits sur la terre, ses membres se sont fait adopter par la mer-mère nourricière jusqu'à en faire partie intégrante et ne plus se distinguer du milieu. Il n'y a pas d'expression particulière signifiant yeux de la mer, masondriake est absent du vocabulaire, comme il y a une expression signifiant dos de la mer dans le vocabulaire vezo, pour la raison que ce sont les Vezo eux-mêmes qui sont les yeux de la mer, masondrano. La mer a un dos, elle a même une bouche et d'autres organes mais n'a pas d'yeux, les hommes pallient ce manque, ils sont le prolongement du milieu, ils en sont l'un des sens.

L'identité n'existe pas indépendamment du groupe, elle est un phénomène social, "l'identité personnelle est inévitablement liée aux autres qui en reconnaissent et en confirment la valeur» (Heribert, 1995). L'individu ne devient pas enfant de la mer automatiquement, il acquiert ce statut lorsqu'il répond à certains critères qui relèvent notamment de la connaissance de la mer, des croyances, des usages et des pratiques qu'elle impose. Le rapport à l'espace est pour les Vezo « un élément fondamental de la symbolique à travers laquelle ont été décrits les rapports sociaux » (Dubourdieu, 1986). La terre est toujours considérée comme la terre des ancêtres, garante de la continuité des générations. Bien que mobile, la société est ancrée en un lieu tanindraza marqué par le hazomanga : pieu commémoratif des sacrifices aux ancêtres ou pilier ancestral.

Figure 1. Le hazomanga Ohimalagne à Andavadoake

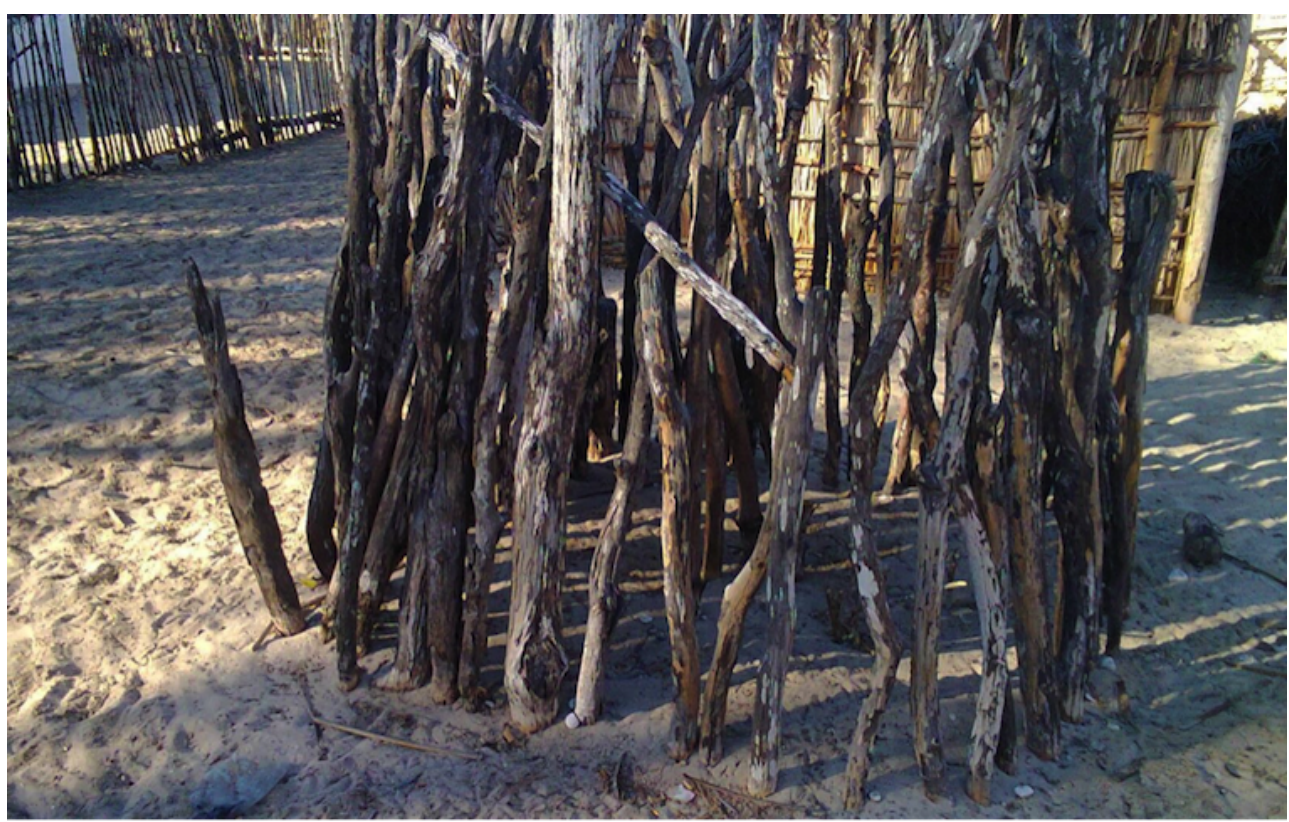

Source : Francis Veriza, 2014.

L'ancrage est toutefois plus ou moins fixe, le hazomanga kombo est hazomanga infirme, qui ne bouge pas; mais le hazomanga peut être mobile mandeha, qui se déplace, qui bouge. Ce hazomanga caractérise l'identité nomade. 
L'espace marin est anthropomorphe, phénomène qui n'est pas spécifique aux Vezo. Les terriens ont également cette représentation de l'espace socialisé. Blanc-Pamard (1995) comme Rakoto Ramiarantsoa (1995) ont mis en évidence la conceptualisation par des agriculteurs de l'Imerina de l'espace paysager en «lieux de corps ». Ils désignent ainsi les différentes parties du paysage "chair de la terre " et "œil de l'eau ", "tête de l'eau», etc. Pour les Vezo, la mer est un être anthropomorphe (Koechlin, 1975; Marikandia, 1988 ; Henry Chartier et Henry, 1998 ; Pascal, 2008).

Tableau 1. Les mots pour dire la mer

\begin{tabular}{|l|l|l|}
\hline Appellation & Traduction & Interprétation \\
\hline Ambavane (Vavarano) & À la bouche (de la mer) & La passe \\
\hline Ambohone & Au dos (de la mer) & Le large \\
\hline Andohariake (Andohariake) & À la tête (de la mer) & Le front récifal \\
\hline Aterano/Amporano & Au cœur/foie (de la mer) & La haute mer \\
\hline Antete & À la tête (la terre en hauteur) & La côte, l'îlot, partie la moins profonde \\
\hline Antsodrano & Au ventre (de la mer) & Le lagon \\
\hline Masondrano & Yeux de la mer & Les Vezo au sens pêcheurs \\
\hline Sonindriake & Lèvre (de la mer) & Le rivage \\
\hline
\end{tabular}

Le vocabulaire traduit le statut de la mer : mère nourricière mais également mère autoritaire. Quand la mer est grosse, elle est en colère, masiake, suite au non-respect des tabous, faly, et des ancêtres. Une cérémonie est alors organisée par les notables, olobe, pour apaiser la colère des ancêtres en leur offrant du rhum rouge et du riz. Riake est un terme spécifique pour désigner la mer. En langue officielle, c'est ranomasina, littéralement « eau salée » ou " eau sacrée ». La sacralité de la mer se trouve dans son rôle purificateur, le bain dans la mer purifie celui qui a commis une faute, il enlève les mauvais sorts. La présence des sites faly interdits en mer atteste également son caractère sacré.

La mer est subdivisée de la côte au large : antete jusqu'au niveau du front récifal avec une levée détritique lohariake ou andohariake (là où les vagues déferlent), c'est le premier secteur que les Vezo dénomment antsodrano (doux et calme). C'est sur les platiers et dans le lagon que se déroulent les différentes activités. Dès que la zone des vagues a franchi ce secteur, les Vezo distinguent ambohone ou ambohondriake, le dos de la mer. La discontinuité du récif, là où la barrière de corail s'interrompt et où il n'y a pas de déferlement de vagues, est appelée ambavane : à sa bouche. En revanche, le cœur ampondriake et le foie aterano se trouvent dans le secteur ambohone. C'est une zone de haute mer surtout fréquentée par les chasseurs de tortues, elle comprend les hautsfonds coralliens qui ne se découvrent pas aux marées basses de vive-eau. Au-delà de ce secteur, l'andrivabe est surtout exploité par les gros bateaux de pêche industrielle. Il n'est plus l'espace des Vezo. 
Figure 2. Séquences paysagères du monde vezo

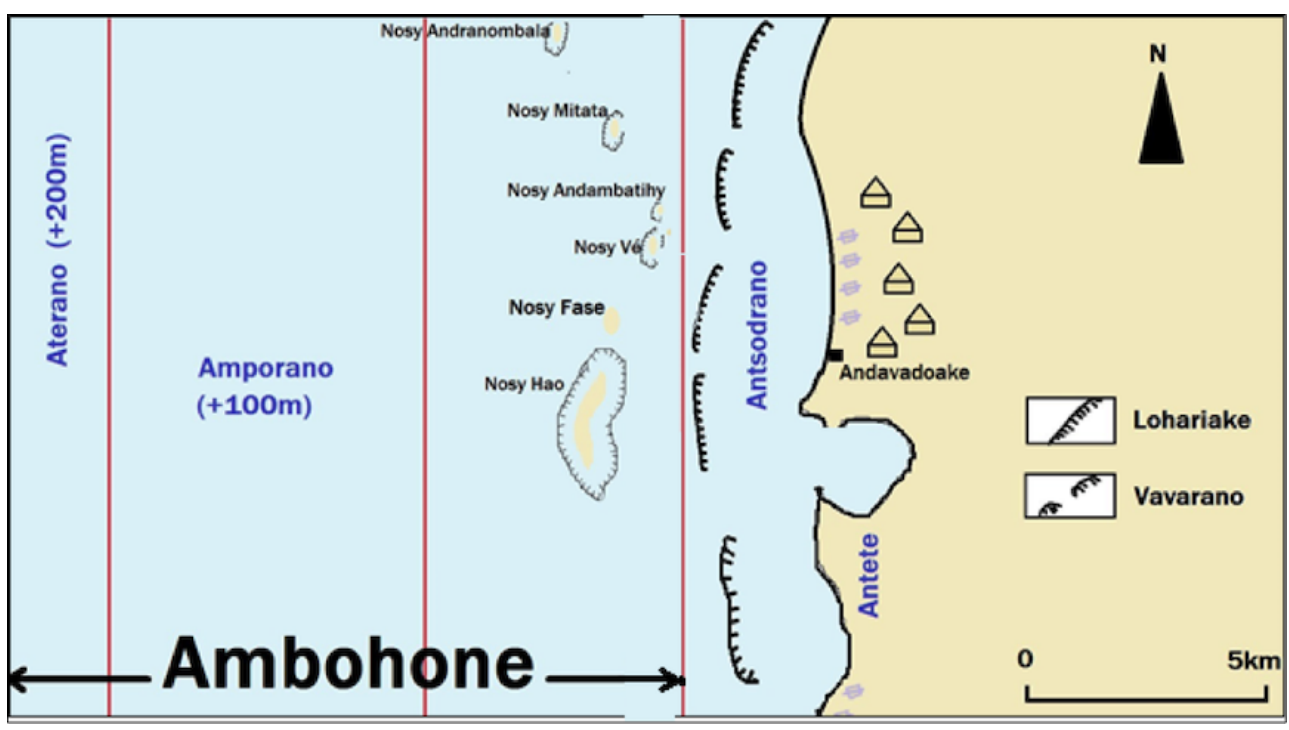

Source : Francis Veriza, 2019

Les Vezo et la mer sont intrinsèquement liés. Les Vezo interprètent le mouvement de la mer comme celui d'un homme: le mauvais temps ou taidraty, nous l'avons vu, est toujours une manifestation de sa colère. La diminution des prises est également inscrite sur ce registre. Le récit d'ampelamananisa, la sirène, ou de bibiandrano, littéralement «bête dans l'eau » ou de beandriake, "grandi en mer » est utilisé pour justifier ce lien d'intimité à la mer. Ces conceptions et pratiques de l'espace sont considérées de manière péjorative dès l'époque coloniale, le projet des colons étant de rationaliser l'exploitation de la ressource. Le développement exogène a des conséquences négatives sur l'état des écosystèmes et conduit, plus tard, à la mise en place des aires protégées.

\section{Développement et conservation : une équation difficile}

Culturellement, les Vezo ne prélèvent que le nécessaire aux besoins quotidiens de la famille, ce qui permet une certaine permanence de la ressource. Ils ont toutefois une capacité d'adaptation qui «s'exprime dans les techniques de pêche très flexibles et toujours prêtes à intégrer de nouvelles données » (Fauroux et al., 1992).

«Les pêcheurs ont su faire évoluer leurs pratiques en fonction des transformations des contextes de production (changements de l'environnement, des prix, des techniques). Les adaptations observées sont tout aussi bien techniques et spatiales que socio-organisationnelles » (Pascal, 2008).

Le discours sur le développement et la sortie de la pauvreté les extrait de leur univers pour les inscrire inexorablement dans une filière dont ils ne constituent désormais que le premier maillon. Dès l'époque coloniale, la science moderne constate un décalage entre le potentiel halieutique du Sud-Ouest malgache et la piètre technicité des pêcheurs vezo (Angot, 1950), qu'il est donc question d'éduquer (Angot, 1961). La décolonisation ne change rien au jugement :

«Les Vezo utilisent des moyens traditionnels peu efficaces tels que les pirogues monoxyles avec balancier, lignes traînantes, lignes à main, sennes de plage 
fabriquées avec des matériaux récupérés sur de vieux pneumatiques, petits filets maillants, barrages et nasses à crevettes » (Couty, 1969). moderne : c'est la promotion de la pêche rationnelle et scientifique. Le Fonds spécial des Nations unies finance en 1968 des projets pour développer la pêche maritime à Madagascar. La Food and Agriculture Organization (FAO) en assure l'exécution en collaboration avec l'Orstom, actuel Institut de recherche pour le développement (IRD). L'objectif est de former des cadres malgaches, d'évaluer les ressources halieutiques, d'étudier les circuits de commercialisation des produits (Couty, 1969). La station marine de Toliara existe depuis 1961, elle est rattachée à l'université de Madagascar jusqu'en 1975, date de la fondation du Centre universitaire régional de Toliara et de l'entrée en vigueur des nouveaux accords de coopération franco-malgaches. Dans les années 1960-1970 sont publiés environ deux cents articles scientifiques et trois thèses d'État (Frontier, 1978). Les pêches sont jugées mal adaptées car pratiquées à l'intérieur du lagon (rapport de l'Unesco/Pnud MAG/88/007 et 507/INT/40-41), les prises ne suffisent pas pour assurer les besoins des exportateurs, la pêche au large devient un défi majeur. Les chercheurs et organismes de développement participent ainsi au changement du paysage vezo en intégrant de nouveaux aspects techniques liés au mode de production. La publication des résultats de recherche incite les entreprises de collecte et d'exportation à s'intéresser à l'exploitation.

«Les langoustes comme les requins ne faisaient pas partie de nos espèces cibles mais nous les avons exploitées sur la demande des collecteurs " (entretien à Andavadoake en 2008).

La promotion des entreprises d'exportation constitue la première motivation des institutions internationales et du gouvernement malgache dans le programme d'amélioration de la pêche. Les organisations de développement proposent successivement des pirogues en fibre de verre et en résine, le moteur en complément de la voile et de la pagaie qui permettent de franchir la barrière de corail pour des prises plus conséquentes. Les instruments du développement sont en partie appropriés mais les pêcheurs craignent de s'aventurer en dehors de leur paysage, constitué à l'intérieur de la barrière de corail. Les dispositifs d'optimisation mal intégrés ont ainsi pour effet la surexploitation du lagon et la dégradation de la ressource qui se traduisent plus tard par la mise en place des dispositifs de conservation. 
Figure 3. Diffusion de la collecte depuis Toliara

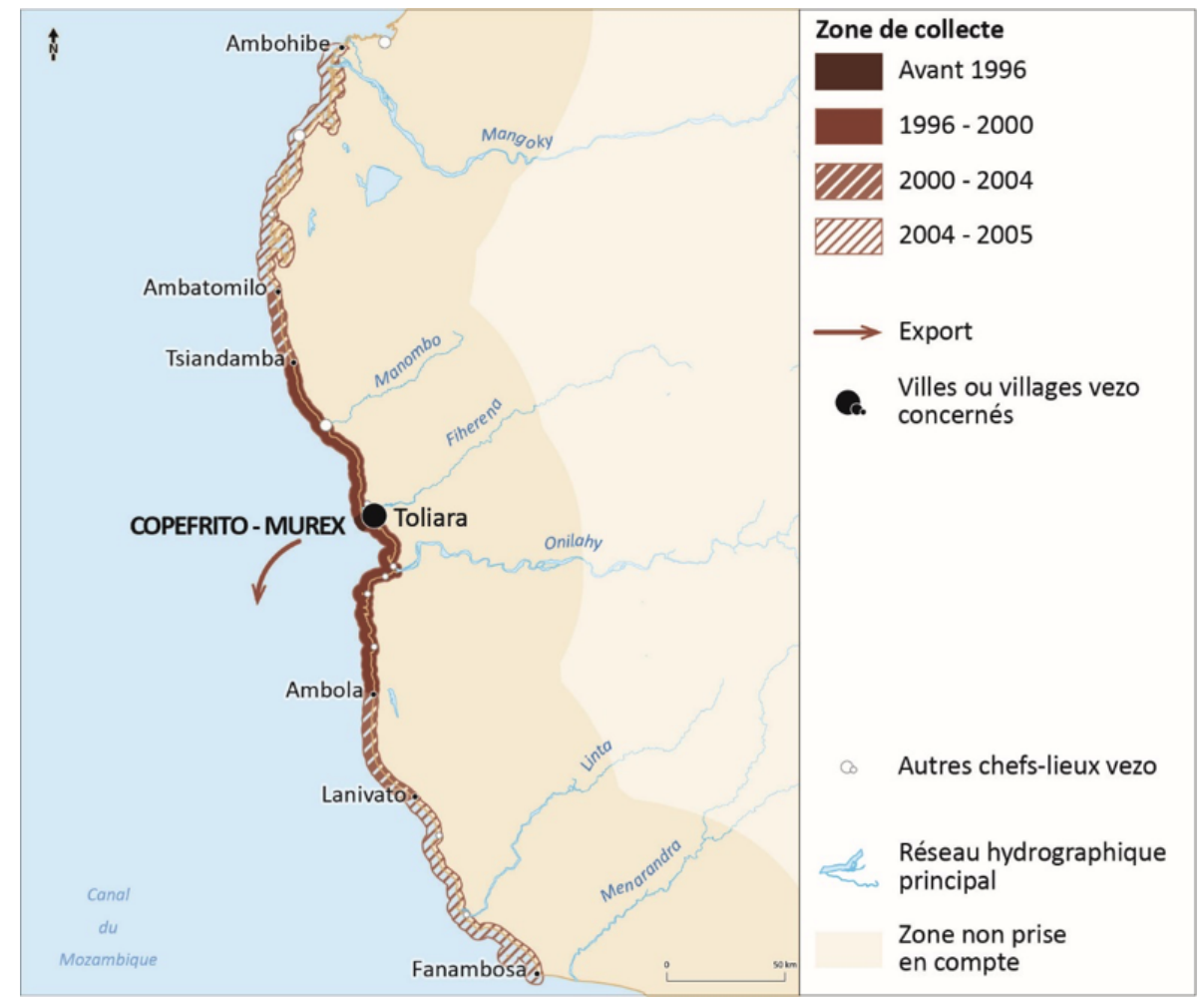

Source : Francis Veriza, 2019.

27 La conservation s'installe mais le principe d'exploitation à l'origine du dérèglement n'est pas remis en cause. Les pêcheurs prélèvent désormais le poisson pour des collecteurs industriels de Toliara. En 2005, tout le littoral est couvert par la collecte qui s'est étendue, depuis la ville capitale du Sud-Ouest jusqu'au Sud et au Nord. En échange des produits de la mer, les collecteurs donnent aux pêcheurs accès à la consommation. La demande accrue en produit halieutique pour l'exportation, ainsi que le bas niveau de rémunération les rendent dépendants de la filière. Le cercle du développement s'établit : la pratique doit gagner en efficacité pour répondre à la double demande de plus de produits pour les maillons en aval et plus de revenus pour les pêcheurs, de manière à accéder aux produits qui leur sont désormais nécessaires. Le filet en Nylon qui a fait son apparition à la fin de la période coloniale voit sa maille se réduire.

«Nous sommes dépendants des collecteurs. L'exploitation d'une telle ou telle espèce est dictée par les collecteurs : on exploite les requins et les concombres de mer car les Chinois nous le demandent. On exploite les poulpes pour la Copefrito et le Murex » (propos d'un sous-collecteur à Andavadoake en 2014).

La capacité d'adaptation des pêcheurs comme la déstructuration des cadres sociaux dans lesquels s'inscrit le rapport à la ressource se traduisent dans les mutations des pratiques: en compensation de l'effort de conservation, les ONG ont par exemple distribué des moustiquaires qui sont transformées en filets dont la maille permet de capturer les plus petits poissons, accélérant la dégradation de la ressource. 
Figure 4. L'utilisation des moustiquaires de la conservation

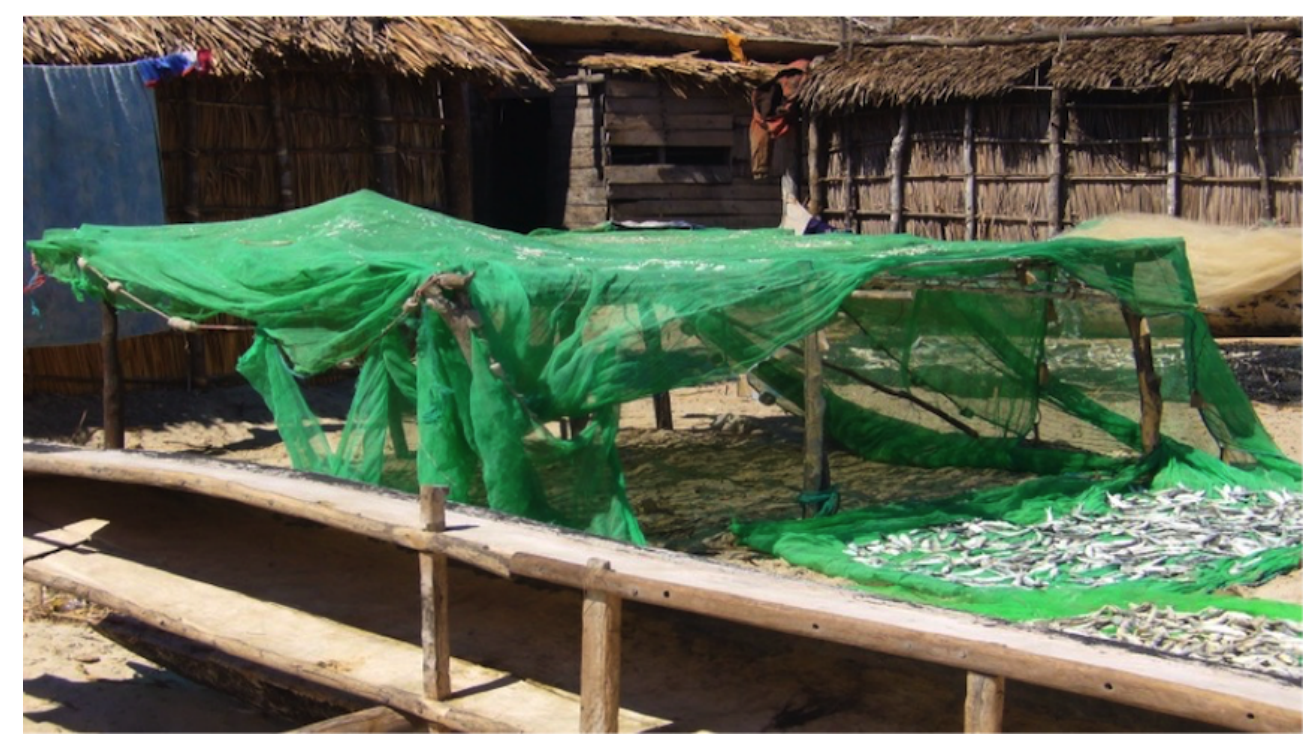

Source : Francis Veriza, 2018.

29 À Madagascar, la nécessité de conservation est un effet du développement (Rodary, 2008). Dans les années 1990, les organisations conservationnistes font le constat alarmant de la dégradation du lagon et de ses ressources, ce qui enclenche un cycle nouveau dans l'intervention extérieure, avec le relais des autorités malgaches. Du Sud au Nord se placent World Wildlife Fund (WWF), Wildlife Conservation Society (WCS), Reef Doctor (RD), Blue Ventures (BV) et Madagascar National Park (MNP), qui sont des organisations de conservation de l'environnement et de développement durable. On observe bien une ingérence écologique au sens de Rossi (2000) afin de mieux contrôler le territoire et ses ressources. 
Figure 5. Le partage de l'espace par les ONG

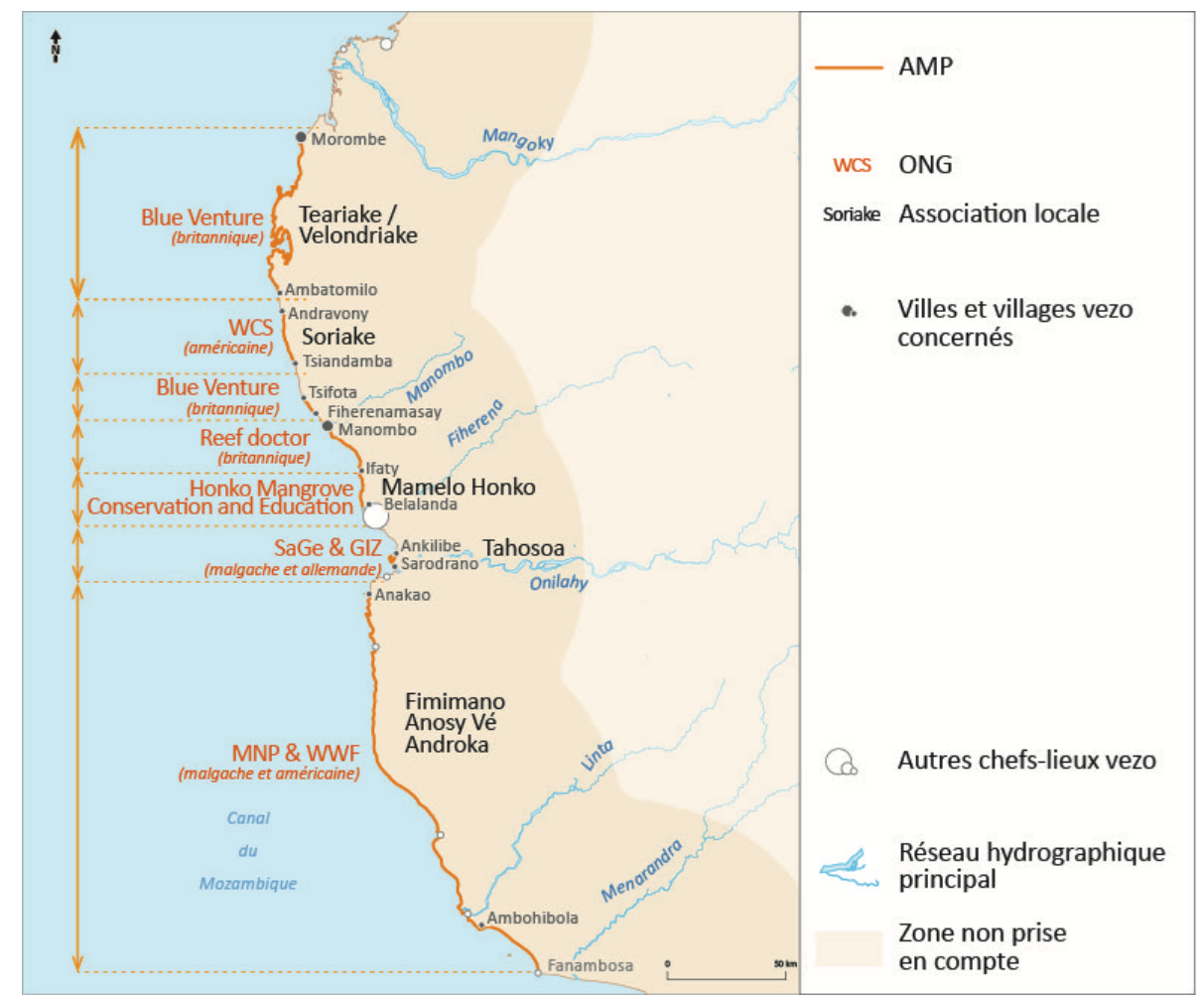

Source : Francis Veriza, 2019.

Le caractère exogène des projets perturbe l'organisation sociale endogène. Cette perturbation est amplifiée par la multitude des interventions qui adoptent des stratégies parfois contradictoires au gré des cahiers des charges soumis par les bailleurs internationaux. Si les pratiques spatiales vezo s'inscrivent dans le mouvement le long de la côte, le partage des zones d'influence des ONG fixe l'action, il la territorialise.

Tableau 2. Relais locaux des organisations internationales

\begin{tabular}{|l|l|l|l|}
\hline ONG & Nationalité & Association locale relais & $\begin{array}{l}\text { Séquence sur le } \\
\text { littoral }\end{array}$ \\
\hline Blue Ventures & Britannique & Teariake/Velondriake & $\begin{array}{l}\text { De Morombe à } \\
\text { Ambatomilo }\end{array}$ \\
\hline WCS & Américaine & Soariake & $\begin{array}{l}\text { Andravone à } \\
\text { Tsandamba }\end{array}$ \\
\hline Blue Ventures & Britannique & Sambele/Mandehasoa & $\begin{array}{l}\text { Tsifota/ } \\
\text { Fieremasay }\end{array}$ \\
\hline Reef Doctor & Britannique & Fimihara & Baie de Ranobe \\
\hline
\end{tabular}




\begin{tabular}{|l|l|l|l|}
\hline $\begin{array}{l}\text { Honko } \begin{array}{r}\text { Mangrove } \\
\text { Education }\end{array} \\
\text { SaGe }\end{array}$ & Belge & Mamelo Honko & Ifaty à Belalanda \\
\hline WWF & Américaine & $\begin{array}{l}\text { Vonihara, Vezo Mitsinjo Ny Hoavy, } \\
\text { Mitsinjo Riake anakao à } \\
\text { Ambohibola } \\
\text { Sarondrano }\end{array}$ \\
\hline MNP & Malgache & Fimimano, Anosy Vé Androka & $\begin{array}{l}\text { Anakao } \\
\text { Ambohibola }\end{array}$ \\
\hline
\end{tabular}

31 Sur les différentes séquences du littoral, le tableau ci-dessus montre les montages entre les ONG internationales de conservation leaders et une myriade de petites structures locales pour la mise en place des aires marines protégées. À Andavadoake, la création de l'association de pêcheurs Velondriake est une condition nécessaire au financement de l'aire marine protégée. L'intervention d'organisations étrangères s'effectue ainsi en lien avec des organisations locales qui légitiment leur présence. Dans la doctrine actuelle du développement durable, il est nécessaire de responsabiliser les usagers, considérés comme les destructeurs de l'environnement (Blanc-Pamard et Boutrais, 2002), de sorte qu'ils s'approprient l'enjeu de la conservation. Les organisations en place s'inscrivent ainsi dans les référents de la démarche ascendante.

" "Community capacity building for Velondriake" narrates the steps taken to achieve this and details the support Blue Ventures has given to the Velondriake community to enable them to realise their vision ${ }^{1}$. »

Dans la conception paysagère développée par Augustin Berque, l'espace ou le paysage de Blue Ventures est débarrassé de sa dimension sensible, ce qui l'oppose à la cosmophanie vezo. Le slogan de l'ONG prête à la communauté ses propres représentations, qu'elle traduit en projet ou en vision.

Le parcours dans lequel s'inscrit la recherche présentée ici a offert l'opportunité de suivre la mise en place des réserves marines depuis son initialisation au début des années 2000 dans une posture d'immersion participante, un des auteurs étant interprète pour une organisation étrangère (Veriza, 2019). Les outils utilisés alors montrent d'emblée la rupture entre les schémas exogènes mobilisés par les ONG et la représentation $\mathrm{du}$ monde que peuvent avoir ses usagers. Sur le registre méthodologique, la cartographie participative est brandie comme la garantie de l'implication locale. Cette pratique est très à la mode en Amérique du Nord et en Europe, elle reprend les codes de représentation cartésienne intelligibles de l'espace qui ne correspondent pas au rapport sensible que les Vezo ont à leur environnement. Nous présentons la cartographie participative mise en place pour l'aire protégée Velondriake. En 2006, les agents de sensibilisation emmènent avec eux des cartes pour présenter aux villageois les zones à préserver. 
Figure 6. Carte « participative » présentée en réunion de concertation à Andavadoake

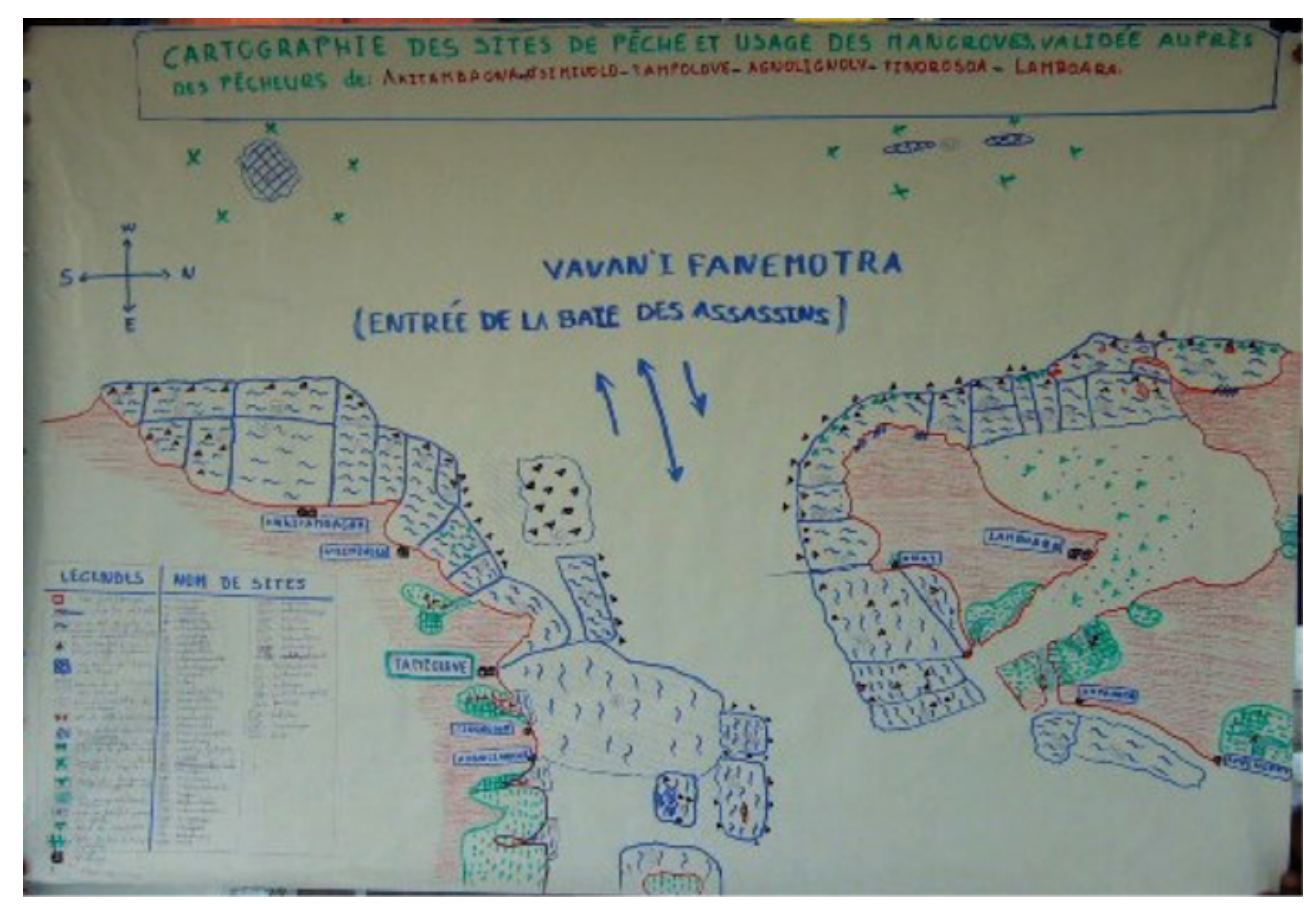

Source : WCS et Blue Ventures, 2006.

Les membres de la communauté, participants du dispositif participatif, ne savent majoritairement ni lire ni écrire. Nous les observons d'emblée perdus par l'orientation de la carte et la position du nord en haut, incapables de positionner dessus leur village et ses environs. Les agents de WCS et BV ne se posent pas la question de leur capacité à comprendre ces référentiels exogènes. Dans leur rapport, les ONG mentionnent des cartes conçues par les villageois eux-mêmes, celle que nous présentons a été maladroitement recopiée par un membre de l'association Velondriake. Ces cartes participatives sont les justificatifs de l'intention de création de réserves par les pêcheurs eux-mêmes. Leur caractère préfabriqué, avec la légende en français et l'indication du nord, traduit des conventions inaccessibles au public auquel elles sont destinées.

\section{Cosmocide?}

La politique de gestion communautaire des écosystèmes développée à Madagascar repose sur des incompréhensions irréductibles et fondatrices, notamment linguistiques et conceptuelles. La manière dont elle est diffusée est contradictoire puisque les opérations de sensibilisation qui visent à établir un consensus s'apparentent à des injonctions : elles négligent les fondements de l'organisation sociale à l'œuvre, leurs réajustements, leurs remaniements, leurs dynamiques. Occidentalo-centrés, les fondements des politiques de gestion communautaire des écosystèmes reposent sur l'illusion d'homogénéité des sociétés qui vivraient dans un espace-temps fixe. La mobilité complique le contrôle.

«L'opposition fondamentale de la mobilité et de la sédentarité structure les savoirs

au point que la conscience de cette donne est effacée. Pourtant, elle est bien 
présente, non seulement dans l'opposition "du" nomade et "du" paysan, mais aussi du marin et du terrien » (Retaillé, 2005). et une rémunération difficiles à concevoir dans d'autres organismes.

Figure 7. Agent de Blue Ventures, Mianatse, expliquant l'importance de la mise en réserve

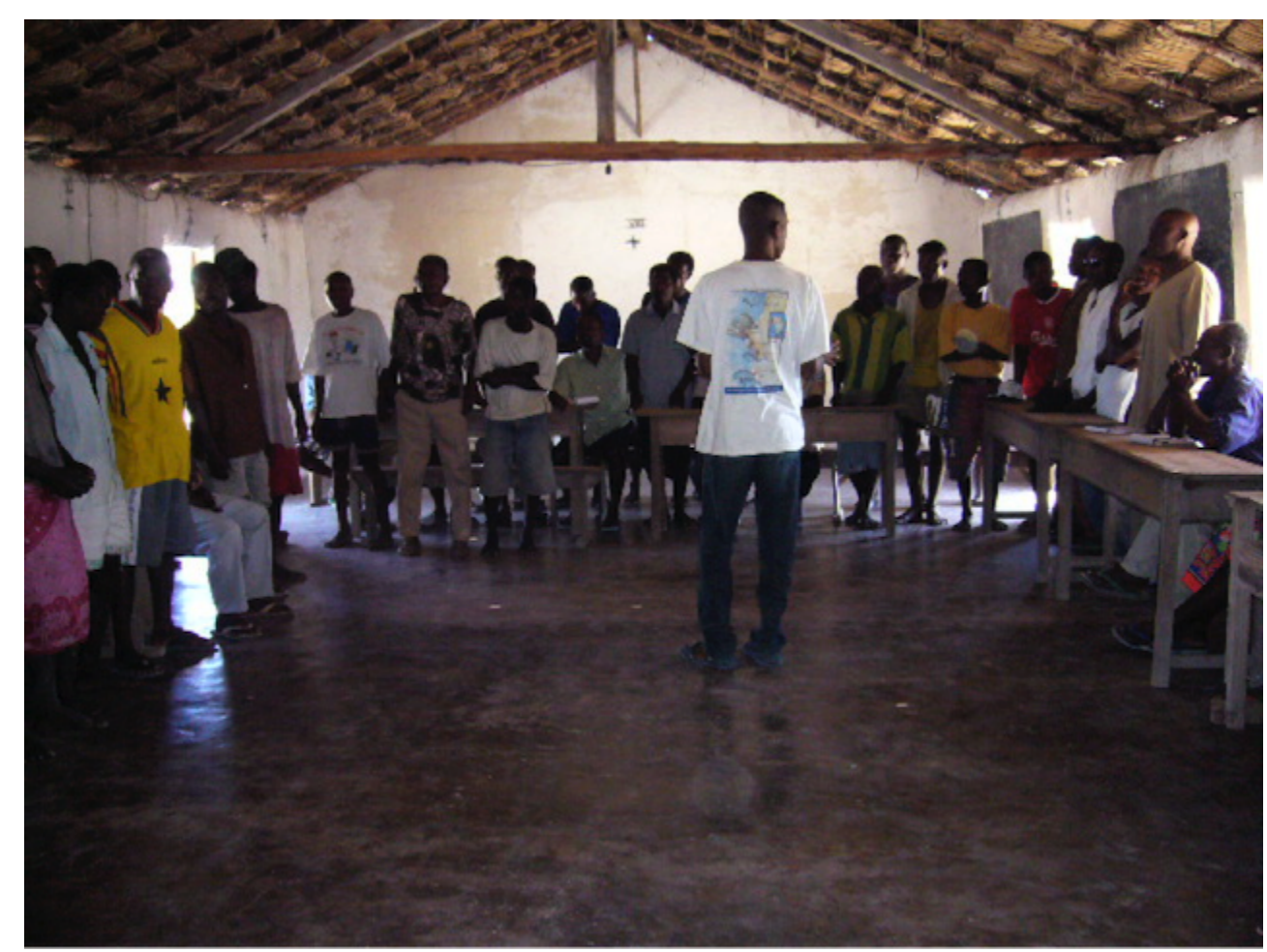

Source : Francis Veriza, 2006.

38 Le retour au village est alors amplificateur des contradictions, les Mianatse se comportant vis-à-vis des ainés en prédicateurs de l'ordre nouveau qui piétinent les valeurs ancestrales.

« Nos aïeux ont pu gérer les ressources en mer sans l'intervention des "Vazaha" ni leur financement. Les interdits suffisaient pour préserver les ressources. Ils nous ont transmis ces savoir-faire d'une génération à une autre. Mais l'arrivée des Vazaha a perturbé la manière dont nous avions géré ces ressources ${ }^{2}$ "(extrait d'un entretien avec un ancien chef de quartier à Andavadoake en 2008).

39 Ce propos concerne les effets du mode de gestion moderne sur la production locale, la disparition des interdits ou leur perte de crédibilité. Le tanifaly, espace interdit, assurait la reconstitution de la ressource et maintenait simultanément l'autorité des olobe et le 
contrôle du monde des ancêtres. En cas de transgression des règles de dimension sacrée, faly, ou de sites sacrés, tanifaly, des sacrifices étaient effectués pour apaiser la colère des ancêtres. Cette coutume est considérée par les Vazaha comme un manque de rigueur et de volonté.

«Ceux qui pensent et exécutent les programmes de conservation tentent d'imposer leurs approches, soit parce qu'ils y croient, soit parce que c'est leur travail. Le décalage entre objectifs et résultats leur apparaît donc comme une incompréhension de la part des locaux: manque d'adhésion de la population; culture traditionnelle peu encline au changement » (Rivière, 2017).

Autour d'Andavadoake, tout l'espace maritime est désormais concerné par l'aire protégée Velondriake.

Figure 8. L'aire marine protégée Velondriake

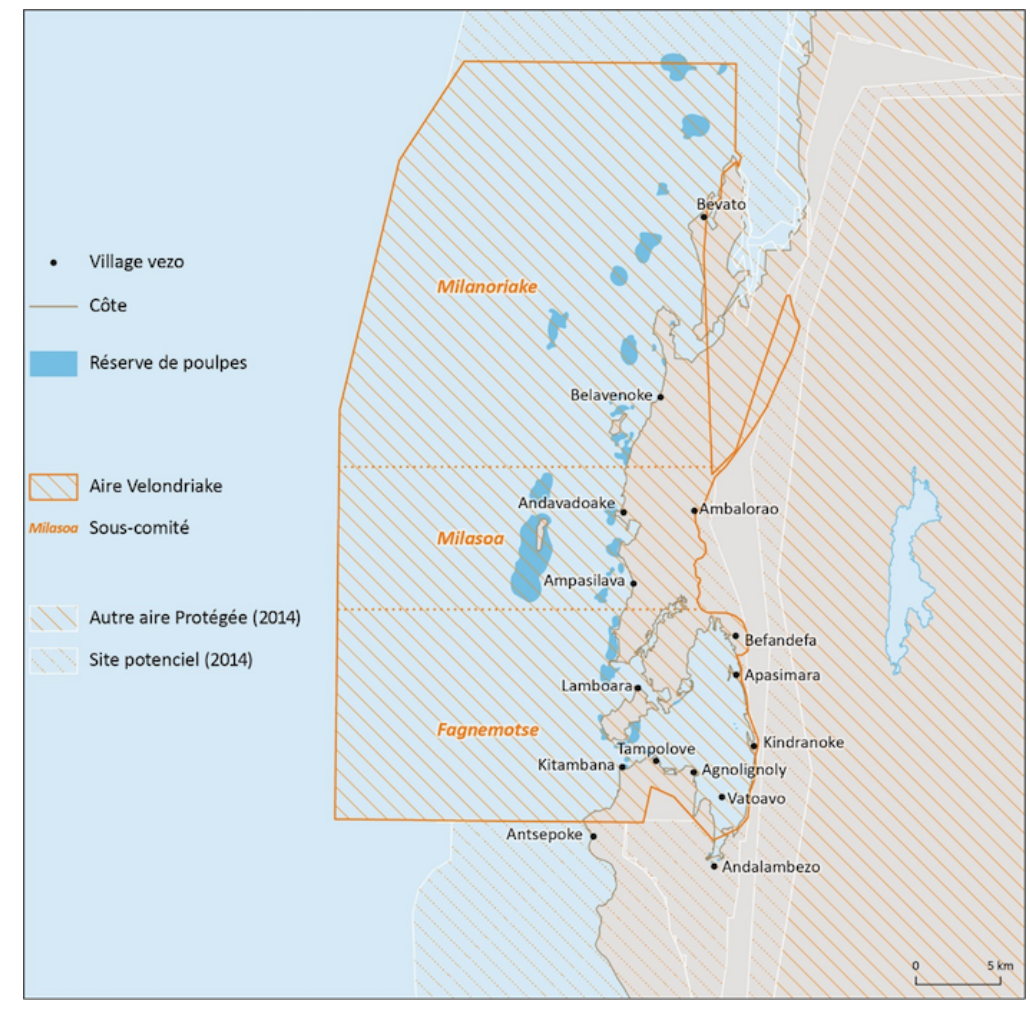

Source : Francis Veriza, 2019.

41 Les pêcheurs vezo reconnaissent aujourd'hui de façon plus ou moins explicite que l'espace marin appartient aux étrangers vazaha. Cette réappropriation se manifeste à travers la délimitation des réserves marines et la mise en place d'un calendrier de fermeture des réserves. Toute la zone de pêche est concernée. La gestion de l'espace marin et l'exploitation des ressources sont devenues l'affaire des étrangers vazaha, les Vezo ne s'en sentent plus les maîtres. Ils sont considérés comme des voleurs quand ils sont surpris dans les réserves. Ils sont pourtant obligés de violer les règles puisqu'ils déclarent ne pas trouver de quoi nourrir leur famille dans les contextes imposés par l'ordre nouveau: mosaré ka mangalats («nous avons faim et c'est pour cela que nous volons »).

L'aire protégée est violée par ceux qui y ont toujours pêché, le tanifaly l'est par des usagers qui ont cessé de craindre les ancêtres, les repères se brouillent. La règle n'est 
plus celle des ancêtres qui sont relayés par les olobe et les gestionnaires du nouveau dina, règlement local, interviennent dans le cadre de l'application de la loi récemment mise en place. Le vieux pêcheur Norbert Alex pourtant membre du comité Velondriake, l'association locale qui gère l'aire marine protégée, précise :

« Nous pouvons toujours négocier en achetant les règles des ancêtres pour lever les interdits. Avec l'actuel dina, c'est-à-dire les règles de fanjakana, nous avons essayé de les acheter mais c'est impossible. Pourtant, dans certains villages, nous avons entendu parler des agents de conservation qui autorisent les usagers à exploiter illicitement les ressources lorsqu'on leur donne de l'argent ${ }^{3}$.»

À l'évocation de la transgression des règles ancestrales auprès d'un olobe, chef de lignage gardien de la tradition, il nous dit :

«Les ancêtres peuvent être indulgents et tolérants envers leurs descendants quand on les respecte mais les étrangers "vazaha" comme l'administration ne comprennent pas la réalité locale.»

De ce fait, les usagers continuent leurs activités informelles au sein même des zones considérées comme interdites. Cela justifie l'expression courante utilisée: «tsy manapadoha ty manenke (on ne risque pas de se faire couper la tête lorsqu'on a dit oui). [Lilindraza] azo vily [...] lily fanjakana tsy azo vily fa laha niazo vily, nivilinay [les règles des ancêtres] on peut les acheter [...] on ne peut pas "acheter" les règles de fanjakana car si on pouvait le faire, nous les aurions achetées" (Pascal, 2008). Les attitudes des villageois envers les projets de conservation sont réfléchies, ils considèrent que leur seul choix est d'accepter. Cette situation les place dans une dépendance contraire aux valeurs vezo.

\section{Conclusion}

45 L'intervention vazaha depuis la précolonisation jusqu'à la mise en place des aires marines protégées marque l'organisation socioculturelle et spatiale vezo: esclavage, évangélisation, colonisation, développement, conservation. Chaque séquence a laissé des traces dans la société et dans le paysage. La société s'est toujours adaptée aux perturbations extérieures, le moteur de l'adaptation était endogène, la mer était le refuge. Avec les aires marines protégées, la pression vient de la mer, le refuge est accaparé.

La communauté a toujours trouvé les moyens d'incorporer les pressions sans céder sur le rapport aux ancêtres, la place des anciens, les interdits. Dans le mouvement actuel, les gardiens de la tradition et de l'ordre ne sont plus craints ni même écoutés, ils disparaissent, la transmission des valeurs s'interrompt. Les ancêtres laissent la place aux opportunismes de nouvelles élites locales qui, pour conquérir leur place dans le jeu, jouent les intermédiaires entre la société et les organismes extérieurs, bradent les savoirs ancestraux pour des injonctions exportées, transgressent les interdits. Des mesures de restriction sont mises en place pour régulariser l'accès aux ressources marines, les connaissances vernaculaires sont marginalisées. Désormais, les structures sociales ne sont plus à l'origine de l'adaptation, il n'y a plus vraiment d'adaptation mais une déstructuration puis quelque chose qui se reconstruit, se reconfigure, qui se restructure mais qui ne correspond plus à la société vezo.

La recherche doctorale qui sert de support à cette contribution ne porte pas sur le paysage. L'opportunité de sa présentation à l'occasion d'un colloque sur les paysages 
marginaux des pays du Sud puis de la contribution à un numéro de la revue Projets de paysage nous a amenés à réinterpréter l'opposition entre les valeurs de la conservation et les valeurs vernaculaires à travers le prisme du paysage. La pensée d'Augustin Berque a servi de cadre théorique à la réexploitation d'un matériau de recherche. La lecture de la cosmophanie vezo et sa confrontation au paysage scientifique moderne à l'origine de l'internationalisation du modèle de conservation renforcent la thèse initiale. L'analyse portait sur la confiscation de ressources au motif de sa conservation, elle nous amène à considérer une décosmisation imposée de l'extérieur, la mise en place d'un modèle incapable de traduire le sens de l'univers dans ce qui entoure les hommes.

\section{BIBLIOGRAPHIE}

Amelot, X. André-Lamat, V., Couderchet, L., « L'espace protégé ou la nature enfermée », dans Laslaz, L., Gauchon, C., Duval, M., Héritier, S. (dir.), Les Espaces protégés, entre conflits et acceptation, Paris, Belin, coll. « Mappemonde », 2014, p. 127-142.

Angot, M., Vie et Économie des mers tropicales : l'exemple malgache, Payot, Paris, Payot, 1961, 326 p.

Angot, M., « Poissons littoraux de Soalara. Carte des fonds, systématique, observations biologiques, possibilités de pêche européenne ", mémoire de l'Institut scientifique de Madagascar, Série A, T. IV, fasc. 1, 1950, p. 175-196.

Berque, A., « Onto/logique du paysage et dépassement de la modernité », conférence inaugurale du colloque international « A qui appartiennent les paysages dans les espaces marginaux des pays du sud ? Mutations contemporaines des territoires, mises en ressource des paysages et recompositions identitaires", Tours, 16 au 18 janvier 2019, p. 10-21.

Berque, A., « Cosmophanie ou paysage », dans Guillaud, D., Seysset, M. et Walter, A. (ed.), Le Voyage inachevé... à Joël Bonnemaison, Paris, Orstom/Prodig, 1998, p. 741-744.

Blanc-Pamard, C et Boutrais, J., « Les temps de l'environnement : d'un sauvetage technique à une gestion locale en Afrique et à Madagascar ", dans Bart, F., Bonvallot, J. et Pourtier, R. (coord.), Regards sur l'Afrique. Historiens et géographes, 2002, p. 249-262.

Blanc-Pamard, C., « Les lieux du corps : l'exemple des communautés rurales des hautes terres malgaches. », dans Claval, P. et Singaravelou (dir.), Ethnogéographies, Paris, L'Harmattan, 1995, p. 51-75.

Cormier-Salem, M.C., « Paysans pêcheurs du terroir et marins-pêcheurs du parcours. Les géographes et l'espace aquatique », L’Espace géographique n 1, 1995, p. 46-59.

Couty, P., « Pêche et commerce des produits de la mer à Madagascar », FAO/Orstom, projet PNUD/FS-Madagascar « Développements des pêches », 1969, 99 p.

Dina, J., «Étrangers et Malgaches dans le Sud-Ouest sakalava (1845-1904) », thèse de doctorat de troisième cycle en histoire, Aix-en-Provence, 1982, 505 p. 
Dubourdieu, L., « Gens de riz dans la basse vallée de la Betsiboka : société sans espace ou espace sans cadres? ", Recherches pour le développement, $\mathrm{n}^{\circ} 2$ 2, 1986, p. 143-193.

Engelvin, A., Les Vezo ou Enfants de la mer. Monographie d'une sous-tribu sakalava à Madagascar, Librairie Vincentienne et Missionnaire, 1937, 169 p.

Fauroux, E., Laroche, J. et Marikandia, M., Brève esquisse d'une description de la société Vezo (littoral occidental de Madagascar) à la fin du Xx $x^{e}$ siècle, Toliara, CNRE/Orstom, 1992, 27 p.

Frontier, S., Activités océanographiques françaises en Océan Indien (Étude du milieu, océanographie biologique et halieutique) de 1966 à 1977, Orstom, 1978, 168 p.

Goedefroit, S. et Razafindralambo, P., « Efflorescence récente du phénomène associatif dans les communautés de pêcheurs traditionnels ", dans Ranaivoson, E. et Zbigniew, K. (ed.), Aménagement de la pêche crevettière à Madagascar, Tananarive, MPRH/FAO, 2001, p. 236-242.

Henry Chartier, C. et Henry, P., « Pratique et perception des espaces aquatiques chez les Vezo de Belo-sur-mer (côte ouest de Madagascar) », Les Cahiers d'outre-mer, vol. 51, n 203, 1998, p. 255-276.

Heribert, A., « Les politiques de l'identité. Nationalisme, patriotisme et multiculturalisme », dans Elbaz, M. et D. Helly, D (dir.), Anthropologie et Société, vol. 19, n 3, Pouvoirs de l'ethnicité, 1995, p. 87-109.

Koechlin, B., Les Vezo du Sud-Ouest de Madagascar : contribution à l'étude de l'écosystème de seminomades marins, Paris, Mouton, École pratique des hautes Études, coll. «Cahiers de l'Homme » 1975, $243 \mathrm{p}$.

Marikandia, M., 1988, « Contribution à la connaissance des Vezo du Sud-ouest de Madagascar : histoire et société de l'espace littoral du Fiherenana au XVIII et au XIX ${ }^{\mathrm{e}}$ siècle », thèse de doctorat, université Paris I, 1988, 481 p.

Pascal, B., « De la « Terre des ancêtres » aux territoires des vivants : Les enjeux locaux de la gouvernance sur le littoral sud-ouest de Madagascar ", thèse de doctorat, Muséum national d'histoire naturelle, IRD, Paris, 2008, 413 p.

Raffestin, C., Pour une géographie du pouvoir, Paris, Libraires techniques, 1980, 249 p.

Rakoto Ramiarantsoa, H., « Regards, ou "c'est en écartant les feuilles que l'on trouve les racines" ", mémoire HDR, université de Paris X, 1995, 161 p.

Razafindrazaka, H., « Le peuplement humain de Madagascar : Anthropologie génétique de trois groupes traditionnels ", thèse de doctorat, 2010, 277 p.

Rejela M., « La pêche traditionnelle Vezo du Sud-Ouest de Madagascar : un système d'exploitation dépassé ? ", thèse de doctorat en géographie, université de Bordeaux 3, 1993, 384 p.

Retaillé, D., «L'espace mobile », dans Antheaume, B. et Giraut, F. (dir.), Le Territoire est mort. Vive les territoires! Une (re)fabrication au nom du développement, Paris, Éditions IRD, coll. « Objectifs Suds», 2005, p. 176-201.

Retaillé, D., « Ethnie ", dans Levy, J. et Lussault, M. (dir.), Dictionnaire de la géographie et de l'espace des sociétés, Paris, Belin, 2003, p. 348-349.

Rivière, M., « Les (dé)connexions du développement. Ethno-géographie systémique de l'aide au développement et à la conservation forestière à Amindrabe, Madagascar ", thèse de doctorat en géographie, université Bordeaux Montaigne, 2017, 469 p. 
Rodary, E., « Développer la conservation ou conserver le développement ? Quelques considérations historiques sur les deux termes et les moyens d'en sortir ", Mondes en développement, $\mathrm{n}^{\circ} 141,2008 / 1$, p. 81-92.

Rossi, G., L'Ingérence écologique. Environnement et développement rural du Nord au Sud, Paris, CNRS Éditions, coll. « Espaces et Milieux », 2000, 246 p.

Veriza, F., «Les yeux de la mer et les médecins de la mer - Des espaces sacrés des ancêtres aux aires marines protégées des Vazaha sur le littoral vezo à Madagascar ", thèse de géographie, universités de Toliara et université Bordeaux Montaigne, 2019, 655 p.

Veriza, F., Chazan-Gillig, S. et Manjakahery, B., « Les Vezo du littoral sud-occidental de Madagascar. Les oubliés de la terre ou les enfants adoptifs de la mer-mère ", Journal des anthropologues, $\mathrm{n}^{\circ}$ 154-155, 2018, p. 261-283, URL : http://journals.openedition.org/jda/7337, DOI : $10.4000 / \mathrm{jda} .7337$.

\section{NOTES}

1. https://blueventures.org/publication/community-creation-and-management-of-thevelondriake-marine-protected-area/

2. «Razanay reo tsy nila jala baka andafy any laha hiaro raha andriake eny io. Faly rey fa ampy niarova aze. Fa fiavia vazaha toa manova raha maro io ".

3. "Zahay afaky mivily lilidraza iny hanafahanay faly iny tsy hahavoandraha gnananay. Fa amy dina noho lalam-panajakana toy zahay la lany ty hainay. Olo reo tsy azo vily fa laha niazo reo ka honivilinay. Mbamy tanà ilany rezao, renay, laha tena manome jala mpiamby reny ka engandrozy hiasa fe mivony ". Propos recueillis à Andavadoake en juillet 2007.

\section{RÉSUMÉS}

La contribution que nous proposons ici est tirée d'une recherche doctorale qui ne place pas le paysage au cœur de ses objets mais étudie l'impact du double mouvement de développement et de conservation sur l'organisation sociospatiale des pêcheurs vezo au sud-ouest de Madagascar. Nous reprenons notre analyse à travers le prisme du paysage, la pensée d'Augustin Berque nous servant de fils conducteurs. L'espace vezo est mobile (Retaillé, 2005), les ingérences extérieures ont tendance à le fixer dans des zonages dont l'aire marine protégée est l'une des dernières manifestations. Après la présentation de l'être au monde vezo puis celle du couple développement-conservation imposé de l'extérieur, nous analysons précisément la mise en place de réserves à Andavadoake par une ONG anglaise et révélons une contradiction entre le monde sensible des pêcheurs et le monde désincarné des scientifiques de la biodiversité. Les Vezo n'ont pas de mot pour dire paysage, nous considérerons donc une cosmophanie (Berque, 1998, 2019). L'observation des transformations sociospatiales prend la forme d'une immersion ethnogéographique et d'entretiens étalés dans le temps qui font apparaître l'établissement des aires marines protégées comme une rupture ontologique. À l'occasion du colloque Aquapa de Tours, en janvier 2019, la présentation de notre sujet à Augustin Berque a amené ce dernier à 
proposer l'idée d'un cosmocide pour décrire l'impact sociospatial des politiques de conservation sur le groupe vezo. Nous étudions cette proposition.

The contribution we propose takes as a starting point doctoral research that does not focus specifically on the landscape but rather on the impact of development and conservation on the social and spatial organization of Vezo fishermen in South-Western Madagascar. In our study we conduct our analysis from the perspective of the landscape and use the thinking of Augustin Berque as a guiding principle. The Vezo space is mobile (Retaillé, 2005) and external interferences have tended to divide it into zones of which a marine reserve is one of the latest illustrations. After the presentation of the Vezo way of life followed by the presentation of the development-conservation approach imposed from the outside, we conduct a detailed analysis of the creation of the nature reserves in Andavadoake by an English NGO and bring to light the contradiction between the sensory world of the fishermen and the intangible world of scientists in biodiversity. Since the Vezos have no word for landscape we consider the notion of a cosmophany (Berque, 1998, 2019). The observation of socio-spatial transformations takes the form of an ethno-geographic immersive study and interviews over time that reveal the establishment of protected marine areas as being an ontological disruption. At the Aquapa conference in Tours in January 2019, following the presentation of our paper, Augustin Berque put forward the notion of cosmocide to describe the socio-spatial impact of conservation policies on the Vezo. We are studying this proposition.

\section{INDEX}

Mots-clés : Madagascar, Vezo, cosmophanie, tanifaly, Velondriake, cosmocide

Keywords : Madagascar, Vezo, cosmophany, tanifaly, Velondriake, cosmocide

\section{AUTEURS}

\section{FRANCIS VERIZA}

Francis Veriza est géographe, docteur des universités de Toliara et de Bordeaux Montaigne, UMR CNRS 5319 Passages et maître assistant, département de géographie/Cedratom de l'université de Toliara. Domaines de recherche : ethnogéographie, développement et conservation veriza7724[at]gmail[dot]com

\section{LAURENT COUDERCHET}

Laurent Couderchet est géographe et professeur des universités, université Bordeaux Montaigne, Passages-UMR 5319 CNRS. Domaines de recherche : géographie de l'environnement et du paysage.

laurent.couderchet[at]u-bordeaux-montaigne[dot]fr 